\title{
Bond energy study of photorefractive properties of doped lithium niobate crystals
}

\author{
Yali He and Dongfeng Xue * \\ State Key Laboratory of Fine Chemicals, Department of Materials Science and \\ Chemical Engineering, Dalian University of Technology, Dalian 116012, P. R. China \\ *Corresponding author. E-mail: dfxue@chem.dlut.edu.cn
}

\section{Supporting Information}

Theoretical classification of PR, ODR and RE ions in terms of $\Delta E_{\mathrm{M}-\mathrm{O}}$ value in the LN crystals (Figure S1). 


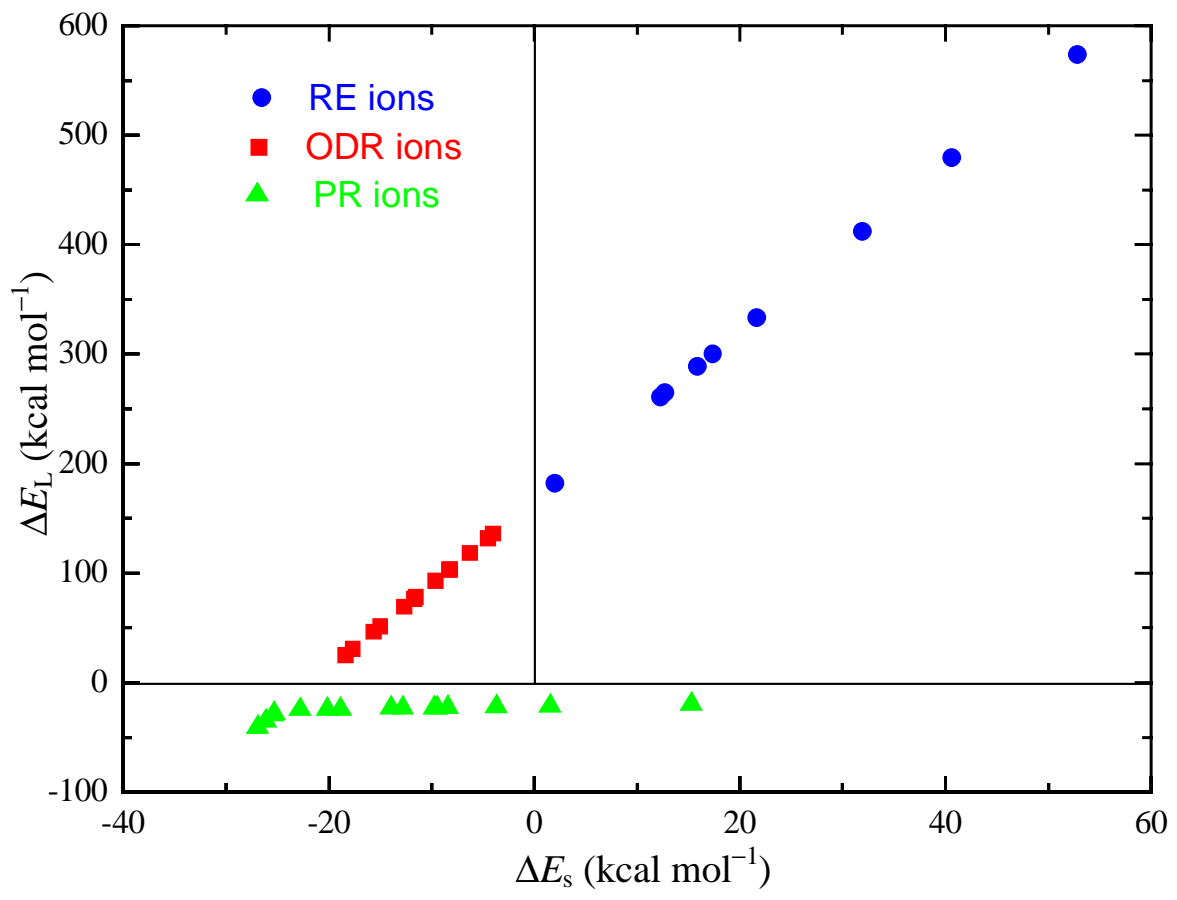

Figure S1 Theoretical classification of various dopants in terms of $\Delta E_{\mathrm{M}-\mathrm{O}}$ value in $\mathrm{LN}$ crystals. Circle symbols correspond to RE ions, square symbols correspond to ODR ions and triangular symbols correspond to PR ions. $\Delta E_{\mathrm{L}}=0 \mathrm{kcal} \mathrm{mol}^{-1}$ is the boundary to separate both PR and ODR ions and $\Delta E_{\mathrm{S}}=0 \mathrm{kcal} \mathrm{mol}^{-1}$ is the boundary to separate both RE and ODR ions. 